\title{
Revisiting Previously Searched Locations in Visual Neglect: Role of Right Parietal and Frontal Lesions in Misjudging Old Locations as New
}

\author{
Sabira K. Mannan ${ }^{1}$, Dominic J. Mort ${ }^{1}$, Tim L. Hodgson ${ }^{1}$, \\ Jon Driver ${ }^{2}$, Christopher Kennard ${ }^{1}$, and Masud Husain ${ }^{1,2}$
}

\begin{abstract}
Right-hemisphere patients with left neglect often demonstrate abnormal visual search, re-examining stimuli to the right while ignoring those to the left. But re-fixations alone do not reveal if patients misjudge whether they have searched a location before. Here, we not only tracked the eye movements of 16 neglect patients during search, but also asked them to click a response button only when they judged they were fixating a target for the very first time. "Re-clicking" on previously found targets would indicate that patients erroneously respond to these as new discoveries. Lesions were mapped with high-resolution MRI. Neglect patients with damage involving the right intraparietal sulcus or right inferior frontal lobe "re-clicked" on previously found targets on the right at a pathological rate, whereas those with medial occipito-temporal
\end{abstract}

lesions did not. For the intraparietal sulcus patients, the probability of erroneous re-clicks on an old target increased with time since first discovering it; whereas for frontal patients it was independent of search time, suggesting different underlying mechanisms in these two types of patient. Re-click deficits correlated with degree of leftward neglect, mainly due to both being severe in intraparietal cases. These results demonstrate that misjudging previously searched locations for new ones can contribute to pathological search in neglect, with potentially different mechanisms being involved in intraparietal versus inferior frontal patients. When combined with a spatial bias to the right, such deficits might explain why many neglect patients often re-examine rightward locations, at the expense of items to their left.

\section{INTRODUCTION}

Patients with neglect often remain unaware of stimuli on their contralesional side. Such neglect is thought to involve pathological biases of spatial attention towards the ipsilesional side of space (Bisiach \& Vallar, 2000; Smania et al., 1998; Kinsbourne, 1993). However, biases in spatial attention may not be the only underlying impairment. Increasingly, the neglect syndrome is considered to consist of a number of component deficits (Husain \& Rorden, 2003; Driver \& Husain, 2002; Bisiach \& Vallar, 2000; Mesulam, 1999; Rafal, 1994), each of which may exacerbate the others, with the exact combination of deficits varying from patient to patient. Some of the component deficits might not in isolation cause neglect, but may nevertheless contribute to the syndrome when combined with other deficits.

One aspect of neglect that has often been observed but which until recently had received relatively little attention is the phenomenon of "revisiting" previously searched locations on the ipsilesional (usually right) side of space. For example, on cancellation tests, where

\footnotetext{
${ }^{1}$ Imperial College London, ${ }^{2}$ University College, London
}

patients are asked to mark each target on a sheet using one mark per target, some patients re-mark targets that they have visibly cancelled before. Such re-marking has sometimes been termed "perseverative," because it might be due to lack of control in preventing recancellation, and consistent with this view, re-marking has been shown to be associated with frontal lesions (Rusconi, Maravita, Bottini, \& Vallar, 2002; Na et al., 1999).

When eye movements are tracked during visual search, many patients with left neglect have also been observed to re-fixate items to the right, recursively searching through items towards that side while continuing to neglect leftward items (Sprenger, Kompf, \& Heide, 2002; Behrmann, Watt, Black, \& Barton, 1997; Chedru, Leblanc, \& Lhermitte, 1973). In principle, such re-fixations could arise for several reasons, and it should be noted that re-fixations may not be equivalent to the "re-marking" phenomena mentioned above, given that no visible mark is made by merely looking at a target.

Zihl and Hebel (1997) first suggested that the large number of re-fixations made by parietal patients (without neglect in their study) might be indicative of a deficit in updating or maintaining locations across saccades. But it could be argued that neglect patients re-fixate 
rightward items because, for them, there is nowhere else to look. That is, they might in principle be perfectly aware that they are re-fixating old previously discovered locations on the right. Alternatively, they may mistakenly judge that they are discovering new targets when actually re-fixating previously found right targets. A critical issue for distinguishing these possibilities is whether or not the patients can discriminate if they are fixating new or old locations during their search.

A recent study introduced a new paradigm precisely in order to assess whether a neglect patient misjudged previously searched rightward locations for novel unsearched locations (Husain, Mannan, et al., 2001). In a computerized search task, target Ts had to be found among Ls (Figure 1) while eye movements were tracked. Critically, the patient was also asked to click on a response button only when fixating a target that was judged to be a new discovery, not when re-fixating an old target that had previously been found and clicked upon. Note that, unlike standard pen-and-paper cancellation tests, but more analogous to search in the real world, the click response did not leave any visible trace, so in this task observers have to remember whether the currently fixated target locations had previously been inspected.

Husain, Mannan, et al. (2001) studied just a single right-hemisphere patient with left neglect in this paradigm, and found that he "re-clicked" at a pathologically high rate on rightward targets when re-fixating those that had previously been found and clicked upon. It was suggested that this tendency to make misjudgments in responses to previously searched locations might contribute to neglect. When combined with any rightward spatial bias in attention, it might exacerbate neglect for leftward items, as the patient would not realize he was repeatedly covering the same ground on the right (Husain, Mannan, et al., 2001).

However, this proposal was based on only a singlecase to date. To investigate it further, the present study applied the new paradigm to 16 neglect patients with middle cerebral artery (MCA) or posterior cerebral artery (PCA) stroke. In addition to assessing how prevalent the "re-click deficit" might be, this study has two important features. First, we performed time-series analyses of search performance, to assess whether any misjudgments in responses to previously found targets depended on the time since a given target had previously been fixated and clicked upon. ${ }^{1}$ Second, we mapped the lesions of our patients using high-resolution MRI (Mort et al., 2003) and reconstructed areas of lesion overlap associated with re-clicking. Taken together, these approaches allowed us to distinguish between two different types of pathological re-clicking. Patients with damage to the right intraparietal sulcus showed re-click behavior that depended on the time since a previously found target had initially been fixated, whereas right inferior frontal patients re-clicked at a pathological rate regardless of the time intervening between initial discovery and re-fixation. These results show that many, but not all, neglect patients misjudge previously found targets as if they were new discoveries, with the deficit and its nature depending on which brain areas are damaged.

\section{RESULTS}

\section{Neglect in the Search Task and Re-fixations}

As expected, neglect patients missed significantly more targets on the left half of the screen (mean 38\%) than the right $[18 \% ; t(15)=2.7, p<.05$; for an example, see Figure 1]. Healthy age-matched volunteers showed equal detection of targets on the left and right sides, missing $16 \%$ on the left and $14 \%$ on the right $[t(14)=$ $0.99, n s]$. Re-fixation rate for each participant is defined as total number of re-fixations/total number of targets fixated, expressed as a percentage. Both patient and
Figure 1. An example of a search array on which are superimposed fixations (dots) and the interpolated scanpath of one neglect patient (Case 15). Note neglect of stimuli on the left and many re-fixations on stimuli to the right.

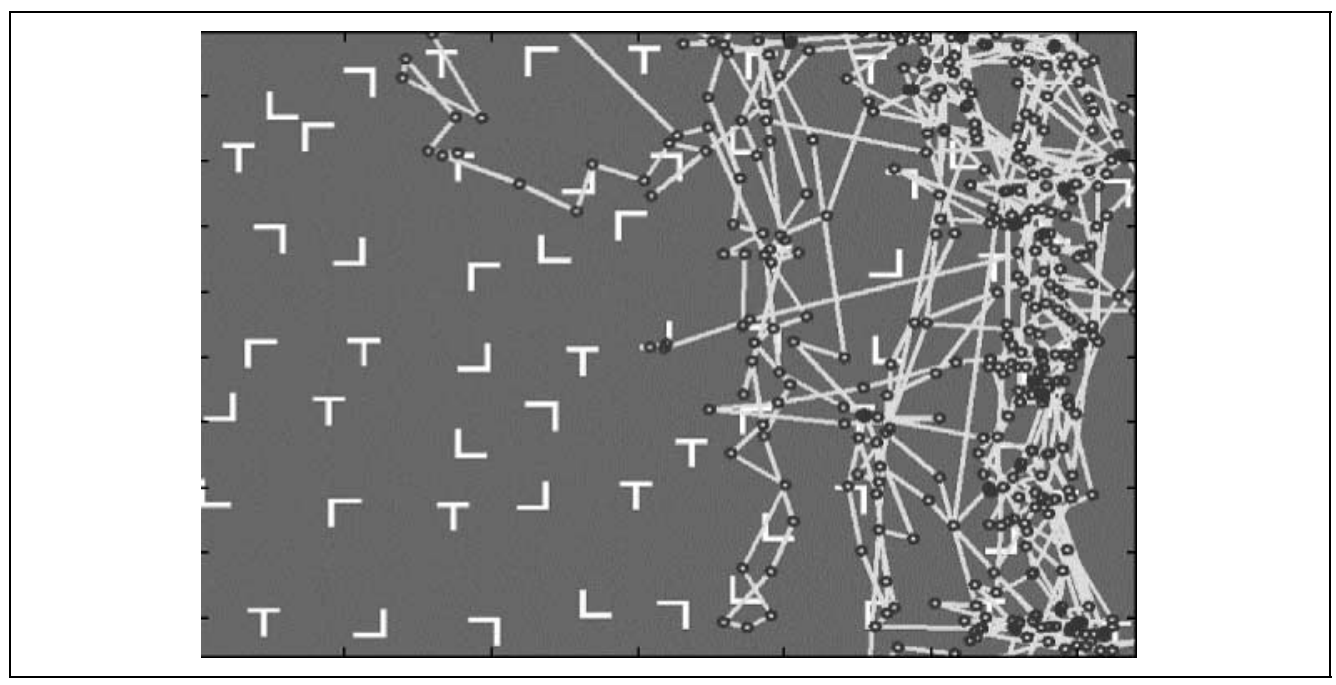


control subjects were more likely to re-fixate targets than distractors (2.8 times and 2.7 times for patients and controls, respectively).

As a group, neglect patients had a significantly higher re-fixation rates (mean $=142 \%$ ) than healthy controls $[40 \% ; t(29)=-3.5, p<.01$; see Figure $2 \mathrm{~A}]$. Ten of the 16 patients had re-fixation rates that fell above the $95 \%$ percentile for controls. The critical issue for this study is whether re-fixations are associated with misjudging previously searched locations for novel ones. This is indexed by the "re-click rate," which indicates any tendency for subjects to erroneously respond to previously found targets as if they were a new discovery.

\section{Re-click Rates}

The re-click rate for each participant is given by total number of re-clicks/total number of targets clicked upon, expressed as a percentage. The mean re-click rate for

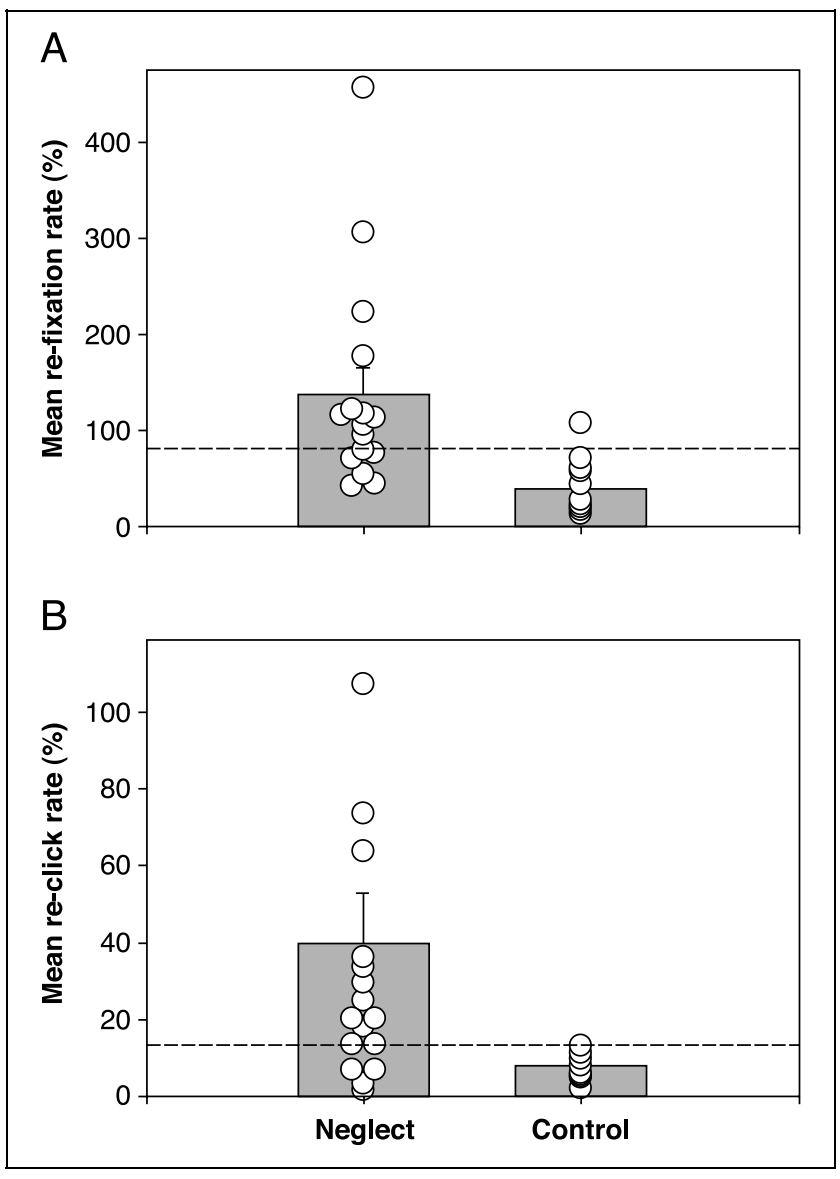

Figure 2. (A) Re-fixation rates for neglect patients and healthy individuals, with individual mean re-fixation rates for each subject shown as circles. Ten of 16 patients had re-fixation rates which fall above the 95th percentile for healthy volunteers, denoted by the dashed line. (B) Re-click rates for neglect patients and healthy volunteers. Ten patients had re-click rates above the 95 th percentile for healthy volunteers. neglect patients (30\%) was significantly higher than for controls $[6 \% ; t(29)=3.1, p<.01]$, who rarely considered a re-fixated target to be a new discovery (Figure 2B). For the neglect group, the probability of re-clicking was highest for targets to the right (Figure 3). In total, 10 of the 16 patients-hereafter termed high re-click grouphad re-click rates beyond the upper 95\% confidence interval for controls (Figure 2B). The remaining $6 \mathrm{pa}-$ tients (low re-click group) had re-click rates within the normal range, demonstrating the re-clicking deficit is not present in all neglect patients. Moreover, this result also shows that brain damage per se does not lead to a pathological re-click rate. Later (in the section on Anatomical Correlates) we show that the deficit cannot also be attributed to lesion size, but rather is associated with damage to specific brain regions.

Importantly, although 7 patients had both a high reclick and a high re-fixation rate, 3 patients had an abnormally high re-fixation rate and a normal re-click rate. Thus, abnormal re-fixation rate alone does not necessarily identify patients who fail to distinguish between novel targets and previously inspected ones. Hence, the importance of measuring re-click rates. Nevertheless, across the group of patients, there was a significant correlation between re-click and re-fixation rates (Figure 4) $[r(15)=.78, p<.0001]$. There was no such correlation between re-fixation and re-click rates for healthy controls $[r(14)=.05, n s]$.

Interestingly, high re-clicking neglect patients not only re-clicked more often on targets they had already clicked upon, but they also more frequently failed to click upon a fixated target they had not previously clicked upon. The high re-click neglect group omitted to click on significantly more newly fixated targets

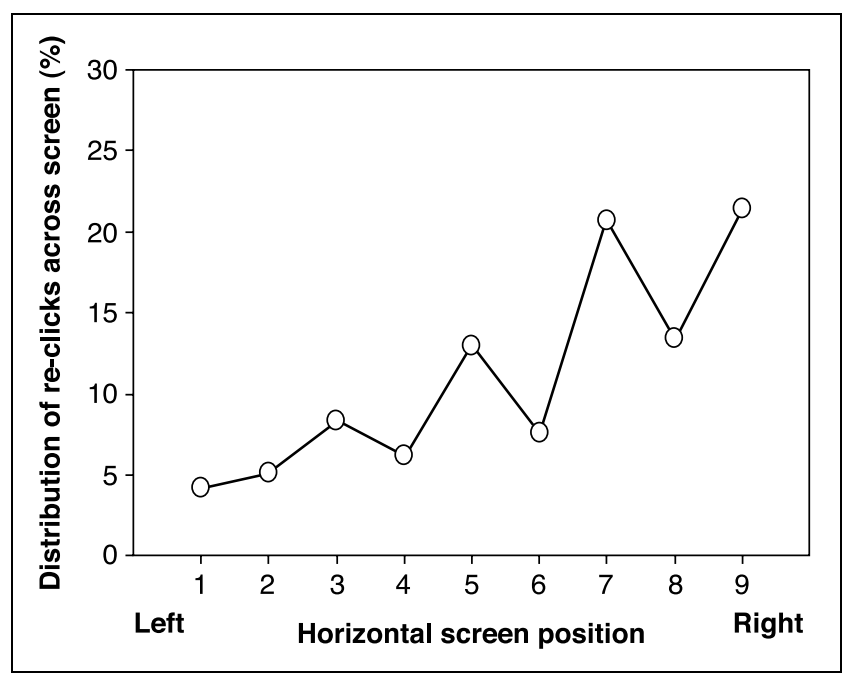

Figure 3. The distribution over space of re-clicks made by neglect patients. The total number of re-clicks made within each virtual column of the screen is expressed as a percentage of the total number of re-clicks made by patients. 


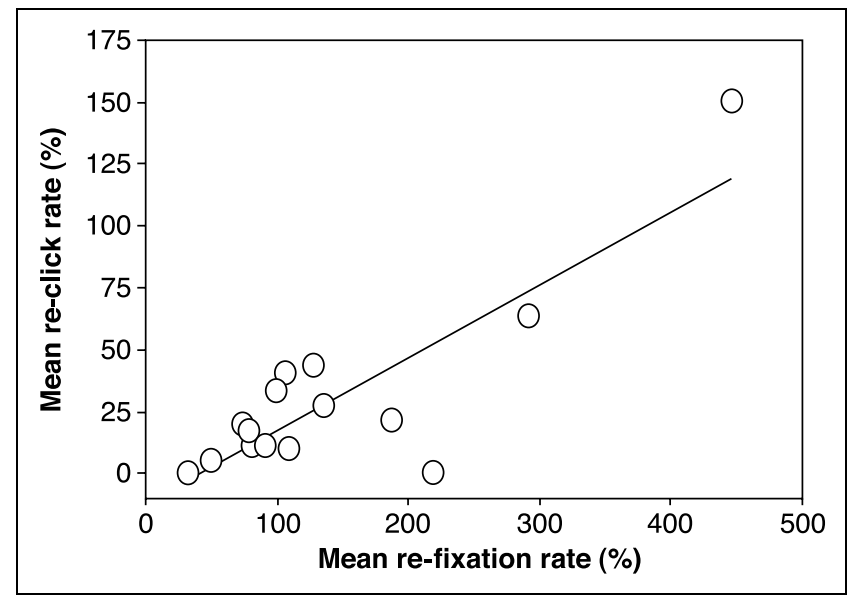

Figure 4. Mean re-click rates plotted against mean re-fixation rates for individual neglect patients. There was a significant correlation $[r(15)=.78, p<.0001]$.

than the low re-click neglect group [mean $=30 \%$ omissions SE 4\% vs. $19 \%$ and 3\%, $t(14)=-2.31, p<$ $.05] .^{2}$ Such an association between these "click omissions" and high re-click rate would not be expected if re-clicking was entirely due to a "perseverative" failure to inhibit responses to rightward targets, because "click omissions" represent failures to respond. Nor would they be explained by items on the right being perceived to be more salient and thus automatically capturing responses.

\section{Anatomical Correlates of High Re-click Rates}

Although 10 neglect patients demonstrated significantly elevated re-click rates, 6 did not. To determine whether key anatomical locations underlie the distinction between these groups, we sought to isolate brain regions that were damaged in neglect patients with high re-click rates, and equally importantly, spared in patients without the deficit. Critically, there was no correlation between lesion volume and re-click rate $[r(13)=-.31, n s]$, or between lesion age and re-click rate $[r(13)=-.30, n s]$. Each patient was assigned to one of two subgroups, according to whether they had frontal involvement or not. Neglect patients with "posterior only" lesions had damage posterior to the central sulcus, with no damage anterior to the precentral sulcus $(n=9)$. Neglect patients with "posterior and anterior" involvement had cortical damage anterior to the central sulcus $(n=5)$; all patients in this subgroup also had some posterior damage.

\section{Parietal Correlate of High Re-click Rate}

Among the 9 patients in the "posterior only" subgroup, 4 had an abnormally high re-click rate: 3 with MCA ter- ritory damage (Cases 5, 6, and 8), and only 1 with PCA damage (Figure 5A). This single PCA patient (Case 2) had damage to white matter deep to the occipitotemporo-parietal junction, in contrast to the other 3 PCA patients whose lesions did not extend so far anteriorly or dorsally. The area of damage common to all 3 MCA patients with abnormally high re-click rates-while also being intact in the 5 posterior subgroup patients with normal re-click rates-was located near the anterior end of the intraparietal sulcus, close to the lateral bank (Figure 5C; Talairach $46-4028$ for centroid; volume $0.58 \mathrm{~cm}^{3}$ ). This area was not simply the most common region of overlap for all $8 \mathrm{MCA}$ patients; that was located more dorsally (Talairach $37-5538)$.

In order to assign statistical significance to the area of overlap, we performed a voxel-by-voxel analysis (Bates et al., 2003). For every voxel that was damaged in at least 3 subjects, we compared the re-click rate of patients with damage to that voxel with those of patients without damage to that particular voxel, producing a $t$ statistic for a given voxel. The area shown in yellow (Figure 5C) yields a value of $t(7)=4.91$, indicating significantly higher re-click rates associated with damage to this region $(p<.002)$. This difference survives Bonferroni correction for multiple comparisons $(p<$ $.05)$, where the number of comparisons was 6 (there were only 6 regions in which the lesions of 3 or more patients overlapped).

An alternative analytical approach is to focus only on patients with relatively small lesions. Seven patients (Cases 4-9 and 15) had focal parietal lesions $<31 \mathrm{~cm}^{3}$; all had posterior lesions except Case 15, who also had involvement anterior to the central sulcus. The maximum area of overlap common to all 4 patients with high re-click rates (Cases 5, 6, 8, 15), which also crucially remained undamaged in the 3 patients with normal reclick rates (Cases 4, 7, 9), again centered on the lateral intraparietal sulcus (Figure 6), slightly more dorsally (Talairach $37-3937$; volume $0.11 \mathrm{~cm}^{3}$ ), and involving a subset of the critical voxels identified in Figure 5 . Note that this overlap region will probably also involve white matter fibers, including those that may connect the parietal cortex to the frontal lobe.

\section{Frontal Correlate of High Re-click Rate}

Four of the five patients in the "posterior plus anterior" neglect subgroup had an abnormally high re-click rate. The area of damage common to all four was in the inferior frontal lobe (Talairach 38, 18, 16 for centroid; $1.78 \mathrm{~cm}^{3}$ ), in a region corresponding to Brodmann's area 44, part of the premotor cortex (Figure 7A). Three of the four posterior plus anterior neglect patients with a pathologically high re-click rate also had damage extending into or bordering the ventrolateral prefrontal cortex. The remaining patient, who had the highest re- 
Figure 5. The

neuroanatomical correlates of the deficit in retaining spatial locations for neglect patients with posterior only (nonfrontal) lesions $(n=9)$. (A) Individual lesions of patients with high re-click rate (patients with MCA damage, Cases 8, 5, 6; patient with PCA damage, Case 2) and overlap map for these

4 patients, where maximum overlap occurs for a small region (shown in green) around the right anterior intraparietal sulcus damaged in the $3 \mathrm{MCA}$ patients. (B) Individual lesions of patients with normal re-click rate (patients with MCA

damage, Cases 9 and 7; patients with PCA damage, Cases 1, 3, 4) and overlap map for these 5 patients, with maximum overlap occurring in the occipital cortex. Importantly, none of the 5 patients with normal re-click rates have damage to the common area of overlap for patients with high re-click rates shown in A. (C) Contrast map highlighting intraparietal locus which is damaged in 3 out of 4 patients with a high re-click rate and intact in the 5 patients with normal re-click rates (shown in yellow). Regions that are damaged in patients with high re-click rates and spared in those patients with normal re-click rates are shown in red to yellow, whereas regions in blue denote areas that are common to patients with

normal re-click rates but spared in patients with high re-click rates.
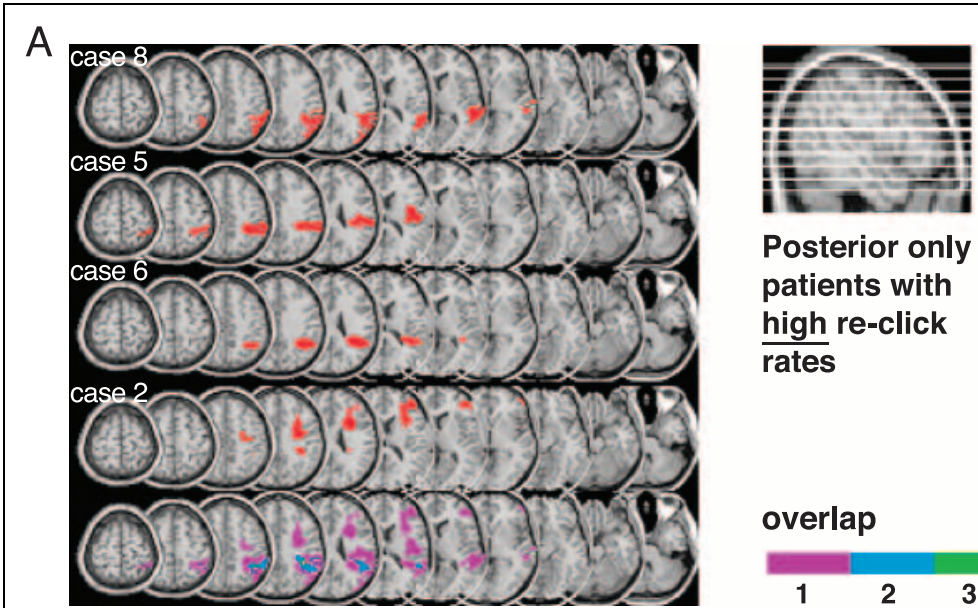

Posterior only patients with high re-click $\overline{\text { rates }}$

\section{overlap}

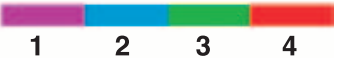

B

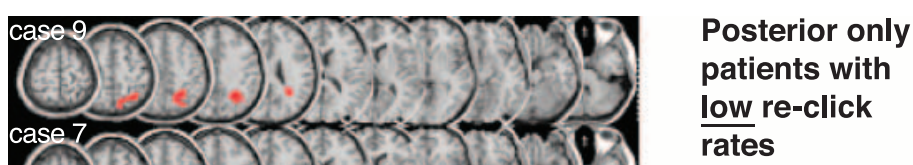

overlap
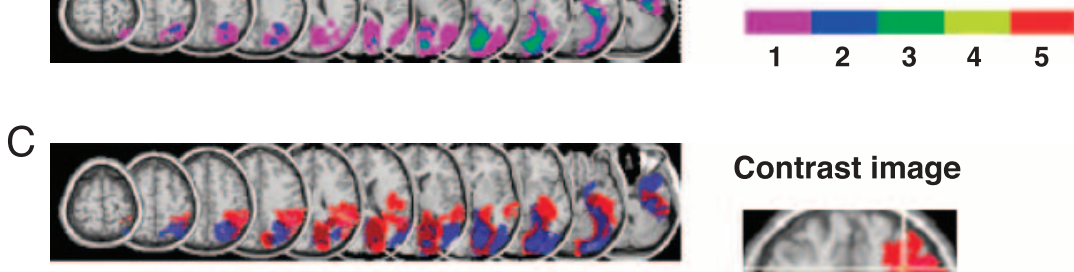

$\begin{array}{lllllllllll}67 & 57 & 47 & 37 & 27 & 17 & 7 & -3 & -13 & -23 & -33\end{array}$
Contrast image

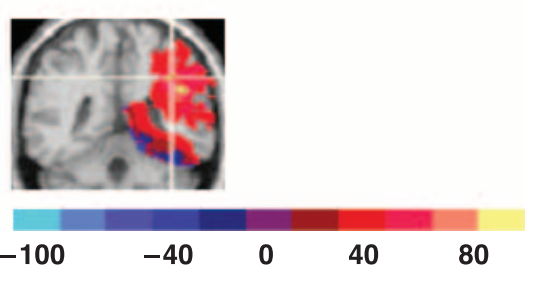

click rate of all patients (Case 15), had damage involving the dorsolateral prefrontal cortex. Only 1 of the 5 posterior plus anterior patients had a normal re-click rate (Figure 7B). Her lesion was posterior to the region of overlap seen in the four frontal patients with abnormally high re-click rates. It involved the posterior premotor cortex and motor cortex, and extended caudally into the inferior parietal lobe. Thus, the subtraction of her lesion from the remaining posterior plus anterior patients with high re-click rates (Figure $7 \mathrm{C}$ ) does not alter their maximum overlap region (displayed in Figure 7A).

\section{Anatomical Correlates of the "Re-fixation" Deficit}

Ten neglect patients had a high re-fixation rate but 6 patients did not. There was no correlation between lesion volume and re-fixation rate $[r(13)=-.22, n s]$, or between age of lesion and re-click rate $[r(13)=$ $-.36, n s]$. Although some patients in either the posterior (Cases 1, 2, 4-8), or frontal group (Cases 11, 13, 15) had high re-fixation rates, other patients with highly similar lesion locations had normal re-fixation rates. As a consequence, voxel-by-voxel analysis (Bates et al., 
Figure 6. The

neuroanatomical correlates of the deficit in retaining spatial locations for neglect patients with focal lesions $(n=7)$. (A) Individual lesions of patients with high re-click rate (all 4 patients have MCA damage) and overlap map for these 4 patients, where maximum overlap occurs for a small region around the right intraparietal sulcus (shown in red) damaged all $4 \mathrm{MCA}$

patients. (B) Individual lesions of patients with normal re-click rate (all patients with PCA damage) and overlap map for these 3 patients, with maximum overlap occurring in the occipital cortex. Importantly, none of these 3 patients with normal re-click rates have damage to the common area of overlap for patients with high re-click rates shown in A. (C) Contrast map highlighting intraparietal locus which is damaged in 4 out of 4 patients with a high re-click rate and intact in the 3 patients with normal re-click rates (shown in yellow). Regions that are damaged in patients with high re-click rates and spared in those patients with normal re-click rates are shown in red to yellow, whereas regions in blue denote areas that are common to patients with normal re-click rates but spared in patients with high re-click rates.

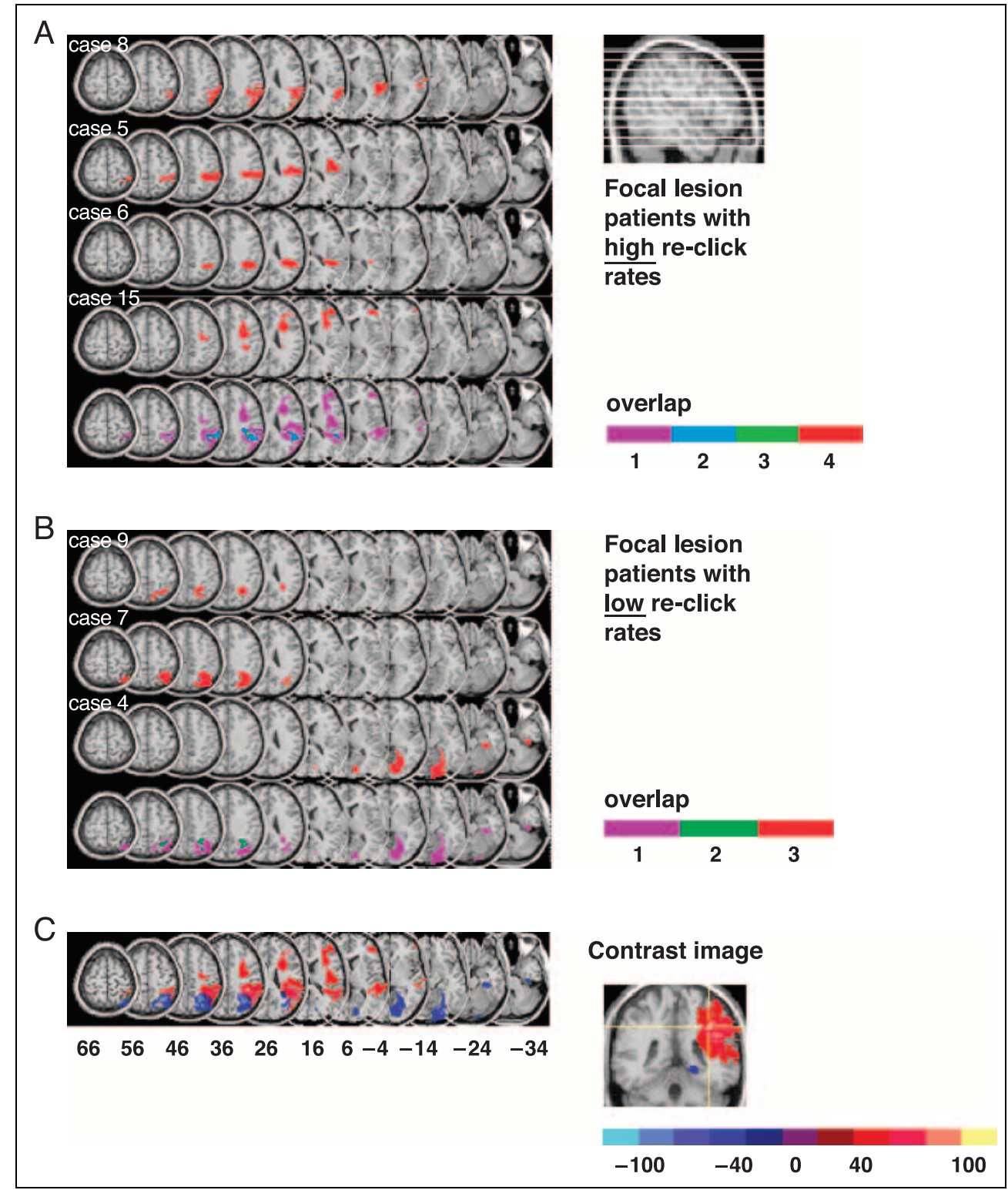

2003) revealed no anatomical specificity associated with high re-fixation rates $[t(7)=0.85, n s]$, unlike that associated with high re-click rates, even when only focal lesions were considered $[t(5)=1.65, n s]$.

\section{Time Interval between Clicking and Re-clicking on a Target}

We next examined the probability of "re-clicking" on a target when re-fixating it, as a function of the time lag or number of intervening saccades between the first click on that target and the subsequent revisit. An increased probability of re-clicking with increasing delay (or intervening number of saccades) would be consistent a failure to keep track of spatial locations during search, whereas a flat or decreasing function might arise from impaired response inhibition ("perseverative" behavior), or even a failure to encode target location. This time-series analysis for the 10 patients with high re-click rates revealed two distinct patterns of behavior. Half the patients demonstrated a significantly increasing probability of misjudging an already found item to be a novel one with increasing delay (Figure 8A) or intervening saccades (Figure 8C), whereas the other 5 patients, had a constant or even decreasing probability (Figure 8B and D).

Importantly, most (4/5) patients in the group with increasing re-click functions had damage to the focal parietal location previously identified in the analysis of patients with focal lesions and high re-click rates (Figure 6C). The other patient was Case 2 who had a large PCA lesion extending to the white matter deep to the occipito-temporo-parietal junction. By contrast, none of the patients with flat/decreasing functions had damage to the key parietal region. Instead, the latter group 
Figure 7. The

neuroanatomical correlates of the re-click deficit in neglect patients with posterior plus anterior lesions $(n=5)$

(A) Individual lesions of patients with high re-click rate (all 4 patients have MCA damage) and overlap map for these 4 patients, where maximum overlap occurs for a region in the right inferior frontal lobe (shown in red) damaged all 4 MCA patients. (B) Individual lesions of a single patient with normal re-click rate. (C) Contrast map highlighting the inferior frontal locus (shown in yellow) which is damaged in 4 out of 4 patients with a high re-click rate and intact in the patient with normal re-click rate. Regions that are damaged in patients with high re-click rates and spared in the patient with normal re-click rate are shown in red to yellow, whereas regions in blue denote areas that are damaged in the patient with normal re-click rates but spared in patients with high re-click rates.

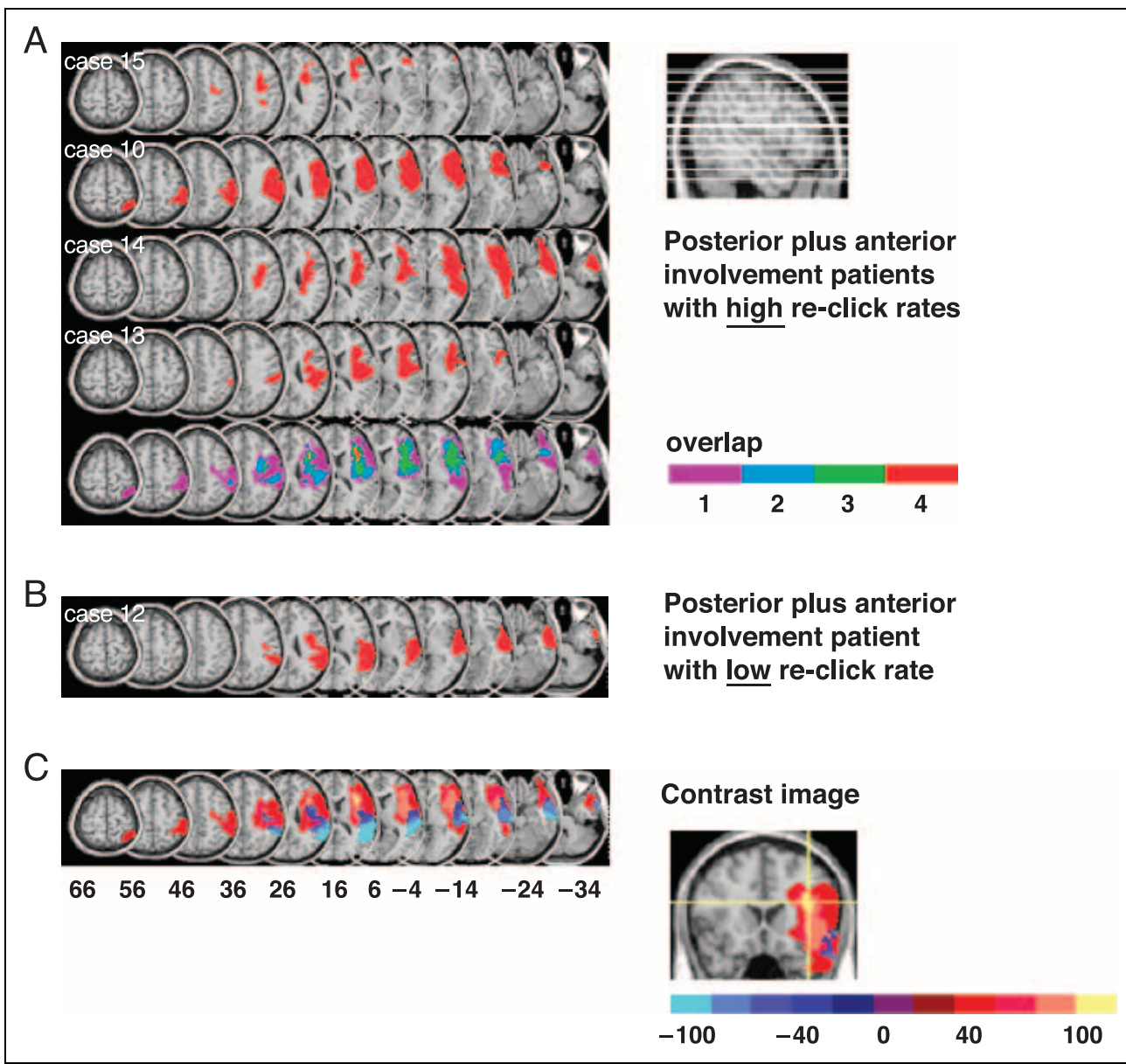

had frontal cortical or subcortical involvement. Thus, increasing re-clicking with time interval was associated with parietal damage, whereas flat re-click functions were associated with frontal/subcortical lesions.

For neglect patients as a whole, the intervals between an initial click on a target and a subsequent re-click were long (mean $=40$ saccades) but much shorter between an initial fixation and a later re-fixation (mean $=5$ ), with this difference being significant $[t(15)=-3.15, p<$ $.01]$. This again emphasizes the dissociation between re-fixations and re-clicks and also demonstrates that reclicking does not occur on most re-fixations.

\section{Relationship of Re-click Rate to Neglect Severity}

We assessed three separate measures of neglect. The first was the difference between miss rates for left versus right targets within the experimental search task itself; the other two measures of neglect were based on the clinical pen-and-paper measures of Mesulam shapecancellation (Mesulam, 1985) and line bisection from the BIT (Wilson, Cockburn, \& Halligan, 1987).

On the experimental search task, there was a moderate but significant correlation between re-click rate and neglect in search across the group $[r(15)=.47, p<.05$;
Figure 9A]. Thus, the greater the re-clicking on rightward items, the greater the severity of leftward neglect. There was also a moderate, but again significant, correlation between re-click rates and the severity of individual patients' clinical neglect as indexed by the cancellation measure $[r(15)=.46, p<.05$; Figure 9B]. These correlations were driven largely by three of the patients identified in our focal lesion analysis (Figure 6A) with involvement of the key parietal region and high reclicking behavior. Their data are shown in filled circles to the right in the graphs displayed in Figure $9 .{ }^{3}$ The correlation between deviation on line bisection and re-click rate was not significant $[r(15)=.13, n s]$. However, one would not predict that a deficit in retaining locations already visited (or response inhibition) would necessarily impair performance on a task such as line bisection.

\section{Effect of Varying Number of Targets}

In 7 patients (Cases 5, 8, 10, 11, 14-16), we also examined the effect of varying the number of targets in the search task. Patients viewed "target only" displays containing either 2, 6, or 19 targets randomly distributed on the screen, without any L-shaped distractors. In- 
Figure 8. The probability of re-clicking or re-fixating on target, as a function of delay between the original click and subsequent re-fixation (for patients with high re-click rates). (A) Probability functions for patients with significant positive slopes. Four patients of these patients have damage to the focal intraparietal locus identified in Figure 6C, whereas the single patient with a PCA lesion and no damage to the critical parietal location, indicated by $\diamond$ symbol, has only a very shallow positive slope. (B) Probability functions for patients with no significant positive slope. None of these 5 patients have damage to the critical intraparietal region. (C) As for A but for delay measured in terms of intervening fixations. (D) As for B but for delay measured in terms of intervening fixations.

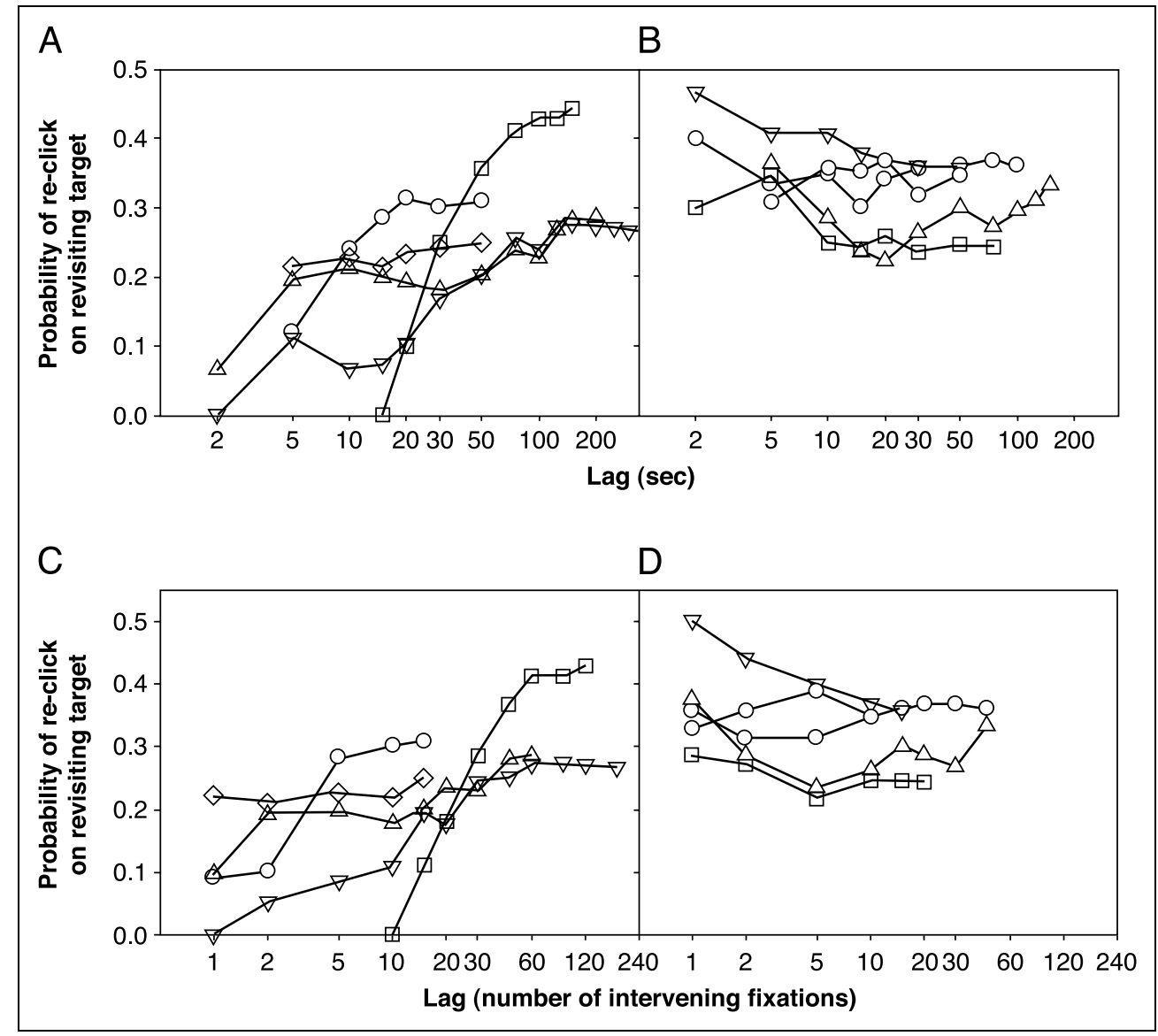

creasing the number of targets led to significantly higher re-click rates (Jonckheere statistic $=133, p<.01$; Figure 10), as well as more severe neglect (Jonckheere statistic $=129, p<.01)$, consistent with the proposal that the mechanisms underlying pathological re-clicking might contribute to neglect behavior. Note that a "perseverative" failure of response inhibition would not on its own easily explain this finding. For the same absolute number of click responses, one would predict a higher re-click rate with smaller set-sizes than with larger ones (see also Husain, Mannan, et al., 2001 for a discussion). Although the number of subjects were small, there was no difference in the performance pattern between the 3 patients who had increasing re-click rate functions in the time-series analysis and the 4 patients with flat or decreasing re-click rate functions.

\section{Varying Perceptual Load during Search}

Can re-click behavior be explained simply by impaired visual perception of targets from discriminators? Neglect patients did find it more difficult than control subjects to select targets from distractors, fixating, on average, 1.9 distractors for every target found [cf. 1.4 for control subjects; $t(29)=4.85, p<.0001]$. Patients also sometimes clicked on distractors (6.4\%) but no more often than controls (6.1\%), and it is possible that this rate is effectively inflated by our strict criterion of considering a click to be a response on the item that is being fixated at the time of the click. (It is likely that sometimes observers click in response to targets they fixated one or more saccades previously.) Re-clicks on distractors were extremely rare (0.6\%; cf. 30\% for targets).

To examine the effect of reduced perceptual load, in 8 patients (Cases 5, 7, 8-10, 13, 1, 4,16), 6 of whom were high re-clickers, we used search displays with salient cross $(+)$ targets rather than Ts; L distractors were unchanged. Patients fixated significantly fewer distractors when searching for cross $(+)$ targets [distractor/ target ratio of 1.34 ; cf. 1.81 for $\mathrm{T}$ targets; $t(7)=6.0, p<$ $.001]$, suggesting they did indeed find target-distractor discrimination easier. Importantly, however, there was no significant difference in re-click rate $[26 \%$ for + targets; cf. $27 \%$ for $\mathrm{T}$ targets; $t(14)=0.41, n s]$ or even in re-fixation rate $[100 \%$ for + targets; cf. $119 \%$ for $t(14)=0.41, n s]$. Thus, these changes in perceptual load did not influence re-click performance.

\section{DISCUSSION}

On this multitarget visual search task, neglect patients missed targets to their left and also re-fixated targets 


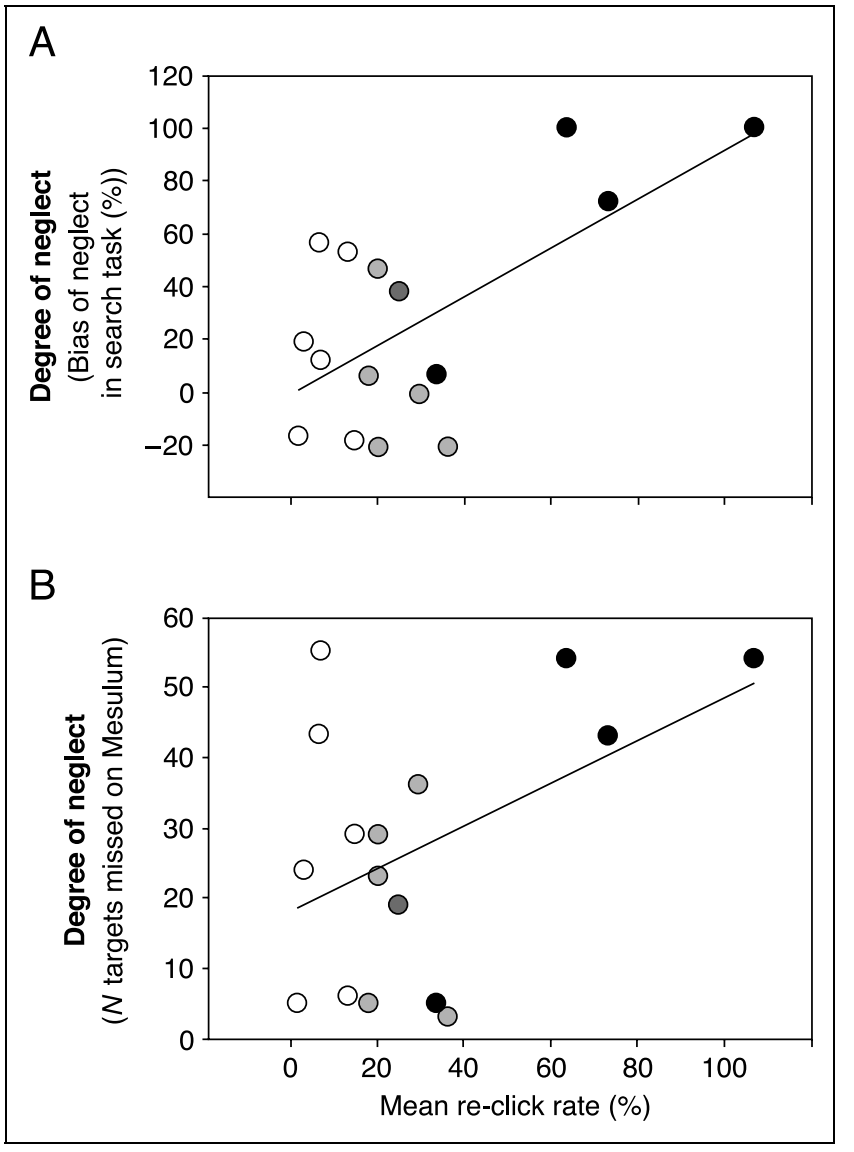

Figure 9. (A) Neglect on experimental search task (19 targets and 44 distractors) plotted against mean re-click rate for individual neglect patients. Percentage neglect was calculated as the difference between the number of targets missed on the left versus right, expressed as a percentage of the total number of targets missed. There was a significant correlation between the degree of neglect and the re-click rate $[r(15)=.465, p<.05]$. Patients with high re-click rates are highlighted by black circles (for patients with damage to the focal parietal location identified in Figure 6C), and light gray circles (for patients with frontal damage) while the single patient with PCA damage and a high re-click rate is indicated by dark gray. (B) The number of targets missed on the Mesulam shape-cancellation task plotted against mean re-click rate for individual neglect patients. Again, there was a significant correlation between the number of targets missed and the mean re-click rate $[r(15)=.456, p<.05]$. Patients with high re-click rates are denoted as in A.

to the right much more often than healthy individuals (Figure 2A). Critically, 10 of the 16 neglect patients reclicked on rightward targets at a significantly higher rate than healthy control subjects (Figure 2B). For 5 of these patients, the probability of re-clicking increased with time (Figure 8A), whereas the other 5 neglect patients with high re-click rates demonstrated a constant or decreasing probability of re-clicking over time (Figure 8C). The remaining 6 neglect patients had re-click rates within the normal range, demonstrating that neither neglect nor brain damage per se leads to a pathological re-click rate.

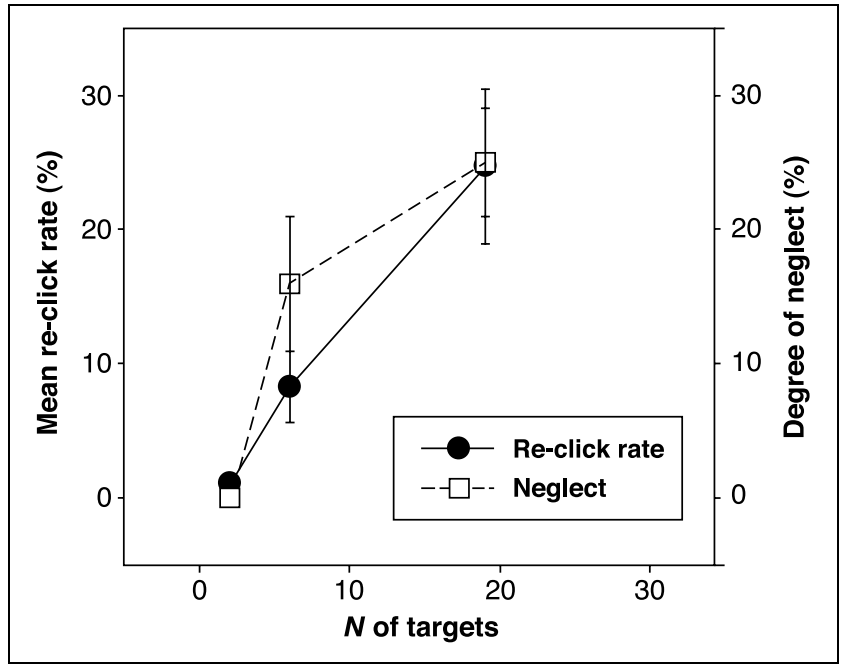

Figure 10. Mean re-click rate (filled circles) and degree of neglect (unfilled squares) increased systematically with increasing numbers of targets in target-only displays.

High-resolution MRI mapping of lesions revealed important anatomical dissociations within the neglect population. Patients with pathological re-click rates had lesions affecting either the right intraparietal sulcus or the inferior frontal lobe; the only exception (Case 2) had a large occipito-temporal lesion that extended deep into the parietal white matter. Neglect patients with normal re-click rates did not have damage to either of the critical locations in the intraparietal sulcus or inferior frontal lobe (Figures 5, 6, and 7). Instead, they had more dorsal lesions (for MCA territory patients), or occipital and medial-temporal damage (for PCA territory lesions), which can also result in neglect (Mort et al., 2003; Cals, Devuyst, Afsar, Karapanayiotides, \& Bogousslavsky, 2002). Importantly, lesion location, rather than the size or age of lesion, was critical to the presence of the reclicking impairment. Across the group of neglect patients, there was a moderate but significant correlation between re-click rate and degree of neglect (Figure 9), driven largely by patients with parietal damage who had both a high re-click rate and also severe neglect, consistent with the proposal that mechanisms underlying this behavior in parietal patients might have a functionally important role in determining the severity of neglect.

\section{Role of Right Intraparietal Sulcus}

Notably, for all patients with damage to the critical locus in the right intraparietal sulcus (which may also involve parieto-frontal white matter fibers), the probability of reclicking on a target increased with time (or intervening saccades) since initial discovery (Figure 8). Because they rarely re-clicked at short intervals-even though the majority of re-fixations occurred during this periodthis pattern of behavior suggests that, for these patients, target locations were actually veridically perceived and 
well encoded into working memory. Furthermore, detailed single-case studies of two parietal patients with high re-click rates (Malhotra, Mannan, Driver, \& Husain, 2004; Husain, Mannan, et al., 2001) demonstrate, using different psychophysical methods, that these patients can accurately encode spatial locations. ${ }^{4}$ Increasing reclicks with time (or intervening saccades) would, however, be consistent with a deficit in keeping track of target locations during extended search.

Several lines of evidence support the proposal that the critical intraparietal sulcus region we have identified may have an important role in the spatial transformations required to keep track of spatial locations across saccades. In rhesus monkeys, neurons within the lateral bank of the intraparietal sulcus (area LIP) encode and "maintain" the remembered locations of saccadic targets over time (Chafee \& Goldman-Rakic, 1998; Mazzoni, Bracewell, Barash, \& Andersen, 1996). Some visually responsive neurons in the LIP also appear to shift their retinotopic representation at the onset of an intended saccade, as if "re-mapping" representations of objects in space to update them across shifts of gaze (Duhamel, Colby, \& Goldberg, 1992).

In humans, this computation has been investigated using the double-step saccade paradigm, where two flashes of light appear in quick succession, such that the second target disappears before a saccade can be made to the first target. Patients with lesions involving the right parietal cortex are impaired at performing this task, suggesting that they are unable to update spatial representations accurately after even one saccade (Heide \& Kompf, 1998; Duhamel et al., 1992). Similarly, transcranial magnetic stimulation of the posterior parietal cortex prior to the second saccade reduces its accuracy (Donkelaar \& Muri, 2002), and a recent study of patients with parietal lesions also implicates this area in the spatial updating of inhibition of return, after a saccade (Sapir, Hayes, Henik, Danzinger, \& Rafal, 2004). Functional imaging studies of double-step (Tobler et al., 2001) and triple-step memory-guided saccades (Heide, Binkofski, et al., 2001) have identified a region within the intraparietal sulcus (Talairach coordinates $40-40$ 44 for Tobler et al. and 44-4836 Heide et al.). This is close to the critical region of lesion overlap we have identified in the posterior neglect patients with high reclick rates (Talairach $37-3937$ ).

We suggest that in our patients with lesions involving the right intraparietal sulcus, uncertainty about locations of previously found targets increases with each saccade. This uncertainty is further exacerbated by interference from newer target locations, leading to increased probability of misjudging old target locations for new as search progresses. Such progressively worsening uncertainty might be due to an accumulating impairment in "re-mapping target locations" across saccades, or impaired "memory" of previously inspected target locations, across saccades. As noted above, there is evidence for both types of signal - re-mapping and maintaining saccadic spatial memory - in the parietal cortex, and it is possible that disruption to both these functions contributes to the re-click deficit we have identified. Certainly, the two potential types of deficit are not mutually incompatible.

A slightly different account of the re-clicking behavior might be framed in terms of the intriguing hypothesis proposed by Manly, Woldt, Watson, and Warburton (2002) to explain visible re-marking on a standard cancellation task. They argue that such behavior is due to patients erroneously transposing leftward stimuli to the right, resulting in apparent re-cancellation. These authors found that removing targets from the left reduced both neglect and re-marking. However, they did not monitor eye movements so it is possible that some targets that are logged as neglected (because they were not cancelled) were nevertheless fixated and therefore form part of the load on memory during search. Furthermore, in our experimental study, subjects with a high re-click rate also failed to click on a higher proportion of fixated targets. Manly et al.'s proposal would not easily account for such "click omissions," because these refer to fixated targets that were not actually responded to.

Finally, it might be argued that increasing re-clicking with search duration reflects a deficit in spatial working memory (SWM) that is independent of saccadic eye movements (i.e., a decay in memory that is not transsaccadic). Because time and number of intervening saccades were not independently manipulated in this unrestricted visual search paradigm, we are not able categorically to distinguish between these possibilities. We note, however, that functional imaging studies of SWM tasks that do not require eye movements often demonstrate activity related to the maintenance of spatial information, which is generally more posterior and dorsal than the parietal locations isolated in this study (D'Esposito et al., 1998). Moreover, recent behavioral data indicate that SWM is more vulnerable to saccadic eye movements than shifts in attention, or limb movements (Pearson \& Sahraie, 2003).

\section{Frontal Lesion Site Associated with High Re-click Rates}

Four out of 5 neglect patients with lesions involving posterior plus anterior damage also showed a high reclick rate, and the area of damage common to these patients centered on the right inferior frontal lobe corresponding to Brodmann's area 44 (Figure 7C). This region has previously been associated with frontal neglect (Husain \& Kennard, 1996) and is considered to be part of the ventral premotor cortex and the homologue of Broca's areas in the left hemisphere (Rizzolatti \& Arbib, 1998; Jackson \& Husain, 1996). In these frontal patients, the probability of re-clicking did 
not increase with time interval between the first click and re-fixation; but rather it stayed constant or declined with increasing delays.

Such behavior might be consistent with a failure to inhibit responses to rightward locations-a type of "perseverative" behavior. Note though that this is not necessarily the same type of perseverative response as seen when some patients with neglect re-mark previously found items on standard cancellation tests (Rusconi et al., 2002; Na et al., 1999). In those cases, targets that are visibly cancelled are often marked again, whereas in our computerized search tasks no marks are visibly left on targets once they have been clicked upon. Moreover, only one of our neglect patients (Case 1) demonstrated re-cancellation of visibly cancelled targets on pen-and-paper tasks, and then only on one occasion. Importantly, this patient actually had a normal re-click rate $(6.7 \%)$ on our task, and his stroke was in the PCA territory sparing both the intraparietal sulcus and frontal lobe.

In support of a role for the right frontal lobe in inhibiting responses to previously found targets, recent imaging studies of response inhibition have highlighted regions of the right inferior frontal gyrus (IFG) very close to the centroid of damage for our frontal patients (38 16 18; cf. 4322 21, Konishi, Kyoichi, Uchida, Sekihara, \& Miyashita, 1998, and 411619 Konishi, Kyoichi, Uchida, Kikyo, et al., 1999). Alternative explanations for the high probability of re-clicking directly after clicking might be that these patients have impaired perceptual discrimination of targets, impaired perceptual localization of targets, or that they encounter difficulty in encoding spatial locations into working memory. Our data are not suggestive of a perceptual discrimination problem as changing the perceptual load did not influence re-click rates. Moreover, the patients still had high re-click rates in the target only conditions (Figure 10), where no perceptual discrimination of targets and distractors was necessary. Poor perceptual localization would seem unlikely given that these patients have frontal lesions. Furthermore, using a vertical SWM task similar to the Corsi block tapping task (Malhotra et al., 2004), we have found that patients with neglect can localize and perceptually encode single target locations as well as controls, and are significantly impaired at remembering two or more locations shown in sequence, consistent with the present observations. Some recent functional imaging data suggest that activation within the prefrontal cortex correlates well with successful encoding of material into working memory (Rypma \& D'Esposito, 2003). Further research is required to distinguish between these possible explanations for the mechanisms underlying the re-click deficit in frontal patients.

The only patient with frontal damage who did re-click more frequently with increasing time had damage centered on the dorsolateral prefrontal cortex (although also encroaching on the IFG). Neurons in the dorsolat- eral prefrontal cortex, like those in the LIP, encode the remembered locations of saccadic targets (GoldmanRakic, 1996). This patient (Case 15), who had the highest re-click rate of all, also had damage to the critical parietal location described above (in contrast to all other frontal patients). Thus, she may have suffered from a combination of underlying deficits.

\section{Re-fixations and Misjudging Previously Inspected Locations during Search}

In this search task, re-fixation and re-click rates were significantly correlated for neglect patients. However, there were important dissociations within individuals: 2 patients with PCA lesions had re-fixation rates of $220 \%$ and $80 \%$, respectively, but both had a re-click rate of only $7 \%$. These are clearly examples of individuals who do not show pathological re-clicking despite having high re-fixation rates. High re-fixation rates may result from a lack of integration of spatial information due to V1 damage (Zihl \& von Cramon, 1985) in our PCA patients, or possibly because of reduced inhibition of return (Bartolomeo, Sieroff, Decaix, \& Chokron, 2001). Whatever the exact cause for dissociations between re-fixations and re-click rates, our data suggest re-fixation does not necessarily index the same underlying deficits as revealed by our re-click measure. High re-fixation rates, unlike high re-click rates, were also not significantly associated with damage to any specific cortical location, again demonstrating a dissociation between re-fixations and pathological re-clicking.

The relationship between re-click rates and neglect is unlikely to be a simple one. In our study, there was a moderate, but significant, correlation between the two measures, suggesting that this association is unlikely to have arisen by chance. However, non-neglect patients can also exhibit a high re-click rate (unpublished observations from our laboratory). Taken together, our results suggest that difficulties in keeping track of previously inspected targets (because of impaired trans-saccadic remapping and/or memory) may be one contributing component to visual neglect, in patients with lesions involving the right intraparietal sulcus. As mentioned, the search task we used differed in one striking aspect from standard cancellation tests: subjects do not visibly mark the targets they visit. Whereas in cancellation tests, patients mark each target they find, which might appear to dispense with any need to remember which locations have been searched before. However, it should be appreciated that in most standard cancellation tests the individual marks made by patients may not be clearly visible in peripheral vision, particularly if neglect patients have a local spatial bias (Rafal, 1994; Robertson, Lamb, \& Knight, 1988) or a restricted field of view (Russell, Malhotra, \& Husain, 2004). Thus, when combined with a rightward attentional bias, keeping track of searched locations may be critical to prevent gaze from 
returning to rightward locations, even if they have been marked before, as the marks may not be visible until gaze returns to them. Consistent with this hypothesis, neglect is greater when cancellation is performed using invisible marks, compared to large and salient red marks that can be seen peripherally (Wojciulik, Husain, Clarke, \& Driver, 2001).

A task like the present search-and-click paradigm may more closely resemble search in the real world than do traditional cancellation tests. When we examine a visual scene, we do not leave marks on each object we examine. Instead, just as in the search task used in this study, we have to keep track of the locations we visit if we are to avoid inefficient re-examination of those locations. Neglect patients with damage to the right intraparietal sulcus appear to be impaired at keeping track of searched locations in this way. When combined with their rightward attentional bias, we propose that such an impairment leads to recursive search of stimuli to the right in experimental search tasks as used here, and also in daily life, where neglect is so apparent despite unlimited viewing time.

The paradigm we have used has the important virtues of allowing patients to explore freely-as in real-world search or clinical cancellation tasks-and relating their re-click behavior directly to the severity of neglect in the same task. But it might be argued that it would be useful also to probe SWM in a more controlled manner, by restricting observers' eye movements to a specific set of locations and probing memory of previously visited places. A recent study, using a gaze-contingent paradigm, has elegantly probed the capacity of spatial memory during search in healthy observers and found evidence for memory for up to four previous locations (McCarley, Wang, Kramer, Irwin, \& Peterson, 2003). Variants of this paradigm may, in the future, be useful to probe the capacity of SWM across saccades in patients with neglect, and also to probe further whether there are differences between neglect patients with parietal and frontal lesions.

\section{METHODS}

\section{Subjects, Imaging, and Lesion Mapping}

Sixteen patients with left neglect following right hemisphere infarction or hemorrhage were recruited. Consent was obtained according to the Declaration of Helsinki, as approved by the hospital ethical committees. Presence of neglect was determined by clinical observation, plus bedside tests including the Mesulam shape-cancellation task (Mesulam, 1985) and line bisection task (Wilson et al., 1987) (see Table 1). Twelve patients had MCA territory strokes; the rest involved only the PCA territory, which can also result in neglect (Cals et al., 2002). Only one neglect patient (Case 1, who did not have frontal involvement) demonstrated re- cancellation of visibly cancelled targets on pen-andpaper tasks, and then only on one occasion. Fifteen age-matched healthy volunteers were controls (mean age $=63, S D=14$ vs. $63, S D=13$ for patients).

All patients had clinical CT scans. In addition, dedicated high-resolution structural MRI scans were also obtained for 14/16 neglect patients. MRI scans were unobtainable for the other two (Cases 11 and 16) because of claustrophobia or loss to follow up. In the MCA neglect group, median time between stroke and scan was 63 days, with the shortest interval being 35 days; for the PCA neglect group, one patient had to be scanned relatively early at 9 days, but the median time was 140 days. MRI scans comprised one 256-slice T1-weighted acquisition (MPRAGE: TR $9.7 \mathrm{msec}$; TE $4 \mathrm{msec}$; TA $12 \mathrm{~min}$ ) performed in the coronal plane aligned perpendicular to the anterior-posterior commissural line, on a Siemens Vision 1.5-T scanner. Withinand between-slice resolution was $1 \mathrm{~mm}$. For each patient, the area of damage was determined by detailed visual inspection of the digital image for every single slice. Lesion delineation was performed by neurologists (authors D. M. and M. H.), by tracing by hand the boundary of the lesion onto every single 1-mm-thick axial slice using a graphics tablet (WACOM Intuos A6). Combining all the slices then led to a 3-D region of interest (ROI) for each patient. For one patient (Case 15), there were two areas of discrete focal damage, one in the frontal cortex and the other in the parietal cortex.

Normalization of each MRI, including the 3-D ROI, to the T1-template was performed with SPM99 (www.fil. ion.ucl.ac.uk/spm). The ROI was used as a mask for the lesion during determination of alignment parameters, to minimize the contribution of abnormal brain to this process (Rorden \& Brett, 2000). The normalized ROI of each neglect patient was superimposed upon the T1 template to find areas of maximal lesion overlap in each group (see Results), as well as lesion volume, using MRIcro shareware (Rorden \& Brett, 2000).

\section{Experimental Procedure}

Stimuli were presented on an SVGA monitor $(29 \times 22$ in. $)$ viewed at $57 \mathrm{~cm}$ distance. Each search display comprised a virtual $9 \times 7$ grid, each cell containing a single $1^{\circ}$ element-either a letter $\mathrm{T}$ (target) or a letter L (distractor) (see Figure 1). The position of each element within each cell was jittered, with 19 targets and 44 distractors randomly distributed over the virtual grid. The number of targets on the right and left halves of the display was balanced across trials. Seven neglect patients (Cases 5, 8, 10, 11, 14-16) were further tested on "Target only" displays, which had varying numbers of Ts (2, 6, or 19), now with no L-shaped distractors. The presentations of these "Target only" screens were interspersed with those of the original search screens, in random order, in the same experimental session. 
Table 1. Patient Details

\begin{tabular}{|c|c|c|c|c|c|c|c|c|}
\hline Case & $\begin{array}{c}\text { Age } \\
\text { (years) }\end{array}$ & Pathology & $\begin{array}{c}\text { Volume } \\
\left(\mathrm{cm}^{3}\right)\end{array}$ & $\begin{array}{c}\text { Age of } \\
\text { Lesion (days) }\end{array}$ & $\begin{array}{c}\text { Visual Field } \\
\text { Defect }\end{array}$ & $\begin{array}{c}\text { Mean Deviation } \\
\text { from Center on } \\
\text { Line Bisection Task } \\
(\mathrm{cm}) \text { (rightward }+ \text { ) }\end{array}$ & $\begin{array}{c}\text { Mesulum Score } \\
\text { (Maximum Possible } \\
\text { Score }=60)\end{array}$ & Lesion Type \\
\hline 1 & 68 & Infarct & 128.2 & 64 & + & 8.67 & 17 & PCA \\
\hline 2 & 76 & Infarct & 152.8 & 227 & + & -0.03 & 41 & PCA \\
\hline 3 & 68 & Infarct & 79 & 207 & + & 2.97 & 36 & PCA \\
\hline 4 & 77 & Infarct & 25.1 & 158 & + & 1.50 & 54 & PCA \\
\hline 5 & 63 & Infarct & 28 & 29 & & 0.33 & 55 & MCA \\
\hline 6 & 59 & Hemorrhage & 17.2 & 7 & + & 3.2 & 6 & MCA \\
\hline 7 & 66 & Hemorrhage & 27.3 & 325 & + & 2.13 & 31 & MCA \\
\hline 8 & 68 & Infarct & 30.9 & 3 & & 2.5 & 17 & MCA \\
\hline 9 & 29 & Infarct & 11.5 & 17 & & 1.13 & 55 & MCA \\
\hline 10 & 70 & Infarct & 133.9 & 324 & & 1.53 & 57 & MCA \\
\hline 11 & 42 & Infarct & - & 11 & & 0.97 & 24 & MCA \\
\hline 12 & 75 & Infarct & 75.7 & 38 & & -0.13 & 4 & MCA \\
\hline 13 & 51 & Infarct & 69.4 & 141 & & -0.83 & 31 & MCA \\
\hline 14 & 59 & Infarct & 114.4 & 356 & + & 2.23 & 37 & MCA \\
\hline 15 & 80 & Infarct & 26.1 & 44 & & 3.5 & 6 & MCA \\
\hline 16 & 64 & Infarct & - & 30 & & 0.33 & 55 & MCA \\
\hline MEAN & 63 & & 66 & 124 & & 1.88 & 33 & \\
\hline$S E$ & 4 & & 13 & 32 & & & 5 & \\
\hline
\end{tabular}

Cases 1-9 had damage to the nonfrontal (posterior only) cortex, whereas Cases 10-15 had both frontal and posterior (posterior and anterior) cortical involvement. Case 16 is a patient with a subcortical lesion. All but 3 of the patients with right MCA infarction had intact visual fields on confrontation. Two of these (Cases 7 and 14) were diagnosed with left homonymous hemianopia, whereas one (Case 6) had a left inferior quandrantic field defect. All patients with PCA territory damage had partial or complete contralesional visual field loss. No patient suffered from dysphasia.

Eye movements were recorded using a video-based pupil tracker with a temporal resolution of 4 msec and spatial accuracy of $<0.5^{\circ}$ (EyeLink; Sensorimotoric Systems, Berlin, Germany). Subjects were asked to keep their head still. The experimenter also monitored gaze position on-line. At the beginning of each session (and after breaks in testing, or following any unintended head movement that disrupted gaze recordings), eye-tracking was re-calibrated. Each trial was initiated by the experimenter and began with a central fixation cross for $1 \mathrm{sec}$, then the search display.

Subjects were instructed to search for Ts (among any Ls). Critically, while looking at any target, they had to click a response button held in the right hand only if they considered this target to be a new discovery (i.e., a target they had not clicked on before). Clicking the response button did not change the display. The response button was held by the patient in a comfortable position, to the right of the midline. Subjects were instructed, and reminded throughout testing, to click on each target found once only. Any later re-click on a previously found target thus indicates a failure to retain the fact that this target had already been discovered. The experimenter terminated each search when the subject indicated verbally that they felt all targets in the display had now been found. Each subject searched a minimum of 5 displays (mean $=7$, range $5-14$ ). Eye movements were analyzed off-line using custom software. An automatic algorithm was used to extract saccades (saccade onset defined as eye speed rising above $25^{\circ} \mathrm{sec}^{-1}$ for two or more samples and a criterion of minimum fixation duration of $60 \mathrm{msec}$ ). Blinks were eliminated from the trace by visual inspection, and saccade and fixation delineation were checked visually in the record. Next, fixations were automatically assigned to items in the display if the mean position fell within $1^{\circ}$ of that item. Two or more consecutive fixations on the same item were re-scored as a single extended fixation. A re-fixation was scored only when at least one fixation elsewhere, on another item, intervened.

Most click responses were made during a fixation, and the few button-clicks occurring during a saccade were 
assigned to the previous fixation. Clicks were assigned to a particular item if eye position at the time of the click was within $2^{\circ}$ of that item; or in the cases where more than one item was within $2^{\circ}$ of current eye position, the click response was simply attributed to the item closest to that eye position.

Button-clicks were assigned to a particular item if eye position at the time of the click was within $2^{\circ}$ of that item. A re-click on an item required at least one fixation on another item between successive clicks (in practice, several fixations elsewhere typically intervened between initial click and later re-click; see Results).

Statistical analyses were performed using SPSS and Sigmaplot (SPSS Science, Chicago, USA); Jonckheere statistic was derived by hand. Statistical significance was set as $p<.05$.

\section{Acknowledgments}

We thank the reviewers of this manuscript and Dr. Andrew Parton for their extremely helpful comments. We thank the patients who participated, Dr. J. Wade, Dr. A. Rudd, and the staff of the stroke units at Charing Cross and St. Thomas' Hospitals. This research was supported by grants from the Wellcome Trust to M. H., C. K., and J. D. J. D. holds a Royal Society Wolfson Research Merit Award.

Reprint requests should be sent to Drs. Sabira Mannan and Masud Husain, Division of Neuroscience and Psychological Medicine, Imperial College London, Charing Cross Campus, London W6 8RF, UK, or via e-mail: s.mannan@imperial.ac.uk orm.husain@imperial.ac.uk.

\section{Notes}

1. We are grateful to one of the reviewers for suggesting this time-series analysis.

2. There was no significant difference between the number of targets fixated between the two groups, so differences in "click omissions" cannot be attributed to the number of fixated targets that had to be kept track of during search.

3. The correlation between these four points alone, in Figure 9A, is high $[r(3)=.81$; cf. $r(15)=.47$ for the whole group].

4. We have also tested 10 neglect patients and 10 control stroke patients on a vertical version of the Corsi blocks task (clinically used to measure spatial working memory capacity). Neglect patients had significantly impaired capacity, but were able to localize single locations well, suggesting that mislocalization is unlikely to account for their deficit on this spatial working memory task.

\section{REFERENCES}

Bartolomeo, P., Sieroff, E., Decaix, C., \& Chokron, S. (2001). Modulating the attentional bias in unilateral neglect: the effects of the strategic set. Experimental Brain Research, 137, 432-444.

Bates, E., Wilson, S. M., Saygin, A. P., Dick, F., Sereno, M. I., Knight, R. T., \& Dronkers, N. F. (2003). Voxel-based lesion-symptom mapping. Nature Neuroscience, 6, 448-450.

Behrmann, M., Watt, S., Black, S. E., \& Barton, J. J. (1997). Impaired visual search in patients with unilateral neglect:
An oculographic analysis. Neuropsychologia, 35, 1445-1458.

Bisiach, E., \& Vallar, G. (2000). Unilateral neglect in humans. In G. Rizzolatti (Ed.), Handbook of neuropsychology (2nd ed., vol. 1, pp. 459-502). Amsterdam: Elsevier.

Cals, N., Devuyst, G., Afsar, N., Karapanayiotides, T., \& Bogousslavsky, J. (2002). Pure superficial posterior cerebral artery territory infarction in The Lausanne Stroke Registry. Journal of Neurology, 249, 855-861.

Chafee, M., \& Goldman-Rakic, P. (1998). Matching patterns of activity in primate prefrontal area $8 \mathrm{a}$ and parietal area 7ip neurons during a spatial working memory task. Journal of Neurophysiology, 79, 2919-2940.

Chedru, F., Leblanc, M., \& Lhermitte, F. (1973). Visual searching in normal and brain-damaged subjects: Contributions to the study of unilateral subjects. Cortex, 9, 94-111.

D'Esposito, M., Aguirre, G. K., Zarahn, E., Ballard, D., Shin, R. K., \& Lease, J. (1998). Functional MRI studies of spatial and nonspatial working memory. Cognitive Brain Research, 7, 1-13.

Donkelaar, P., \& Muri, R. (2002). Craniotopic updating of visual space across saccades in the human posterior parietal cortex. Proceedings of the Royal Society London B, 269, 735-739.

Driver, J., \& Husain, M. (2002). The role of spatial working memory deficits in pathological search by neglect patients. In the cognitive and neural bases of spatial neglect. In H.-O. Karnath, A. D. Milner, \& G. Vallar (Eds.), The cognitive and neural bases of spatial neglect. Oxford: Oxford University Press.

Duhamel, J. R., Colby, C., \& Goldberg, M. E. (1992). The updating of the representation of visual space in parietal cortex by intended eye movements. Science, 255, 90-92.

Goldman-Rakic, P. S. (1996). The prefrontal landscapeImplications of functional architecture for understanding human mentation and the central executive. Philosophical Transactions of The Royal Society of London. Series B, Biological Sciences, 351, 1445-1453.

Heide, W., Binkofski, F., Seitz, R. J., Posse, S., Nitschke, M. F., Freund, H. J., \& Kompf, D. (2001). Activation of frontoparietal cortices during memorized triple-step sequences of saccadic eye movements: An fMRI study. European Journal of Neuroscience, 13, 1177-1189.

Heide, W., \& Kompf, D. (1998). Combined deficits of saccades and visuo-spatial orientation after cortical lesions. Experimental Brain Research, 123, 164-171.

Husain, M., \& Kennard, C. (1996). Visual neglect associated with frontal lobe infarction. Journal of Neurology, 243, 652-657.

Husain, M., Mannan, S., Hodgson, T., Wojciulik, E., Driver, J., \& Kennard, C. (2001). Impaired spatial working memory across saccades contributes to abnormal search in parietal neglect. Brain, 121, 941-952.

Husain, M., \& Rorden, C. (2003). Non-spatially lateralized mechanisms in hemispatial neglect. Nature Reviews: Neuroscience, 4, 23-36.

Jackson, S. R., \& Husain, M. (1996). Visuomotor functions of the lateral pre-motor cortex. Current Opinion in Neurobiology, 6, 788-795.

Kinsbourne, M. (1993). Orientational bias model of unilateral neglect: Evidence from attentional gradients within hemispace. In I. H. Robertson \& J. C. Marshall (Eds.), Unilateral neglect: Clinical and experimental studies (pp. 63-86). Hove: Erlbaum.

Konishi, S., Kyoichi, N., Uchida, I., Kikyo, H., Kameyama, M., \& Miyauchi, S. (1999). Common inhibitory mechanism in 
human inferior prefrontal cortex revealed by event-related functional MRI. Brain, 122, 981-991.

Konishi, S., Kyoichi, N., Uchida, I., Sekihara, K., \& Miyashita, Y. (1998). No-go dominant brain activity in human inferior prefrontal cortex revealed by functional magnetic resonance imaging. European Journal of Neuroscience, 10, 1209-1213.

Malhotra, P., Mannan, S., Driver, J., \& Husain, M. (2004). Impaired spatial working memory: One component of the visual neglect syndrome? Cortex, 40, 667-676.

Manly, T., Woldt, K., Watson, P., \& Warburton, E. (2002). Is motor perseveration in unilateral neglect "driven" by the presence of neglected left-sided stimuli? Neuropsychologia, 40, 1794-1803.

Mazzoni, P., Bracewell, R., Barash, S., \& Andersen, R. (1996). Spatially tuned auditory responses in area LIP of macaques performing delayed memory saccades to acoustic targets. Journal of Neurophysiology, 75, 1233-1241.

McCarley, J., Wang, R., Kramer, A., Irwin, D., \& Peterson, M. (2003). How much memory does oculomotor search have? Psychological Science, 14, 422-426.

Mesulam, M.-M. (1985). Principles of behavioural neurology. Tests of directed attention and memory. Philadelphia: Davis.

Mesulam, M. M. (1999). Spatial attention and neglect: Parietal, frontal and cingulate contributions to the mental representation and attentional targeting of salient extrapersonal events. Philosophical Transactions of the Royal Society of London. Series B, Biological Sciences, 354, 1325-1346.

Mort, D., Malhotra, P., Mannan, S., Rorden, C., Pambakian, A., Kennard, C., \& Husain, M. (2003). The anatomy of visual neglect. Brain, 126, 1986-1997.

Na, D. L., Adair, J. C., Kang, Y., Chung, C. S., Lee, K. H., \& Heilman, K. M. (1999). Motor perseverative behaviour on a line cancellation task. Neurology, 52, 1569-1576.

Pearson, D. G., \& Sahraie, A. (2003). Oculomotor control and the maintenance of spatially and temporally distributed events in visuo-spatial working memory. Quarterly Journal of Experimental Psychology, 56A, 1089-1111.

Rafal, R. (1994). Neglect. Current Opinion in Neurobiology, 4, 231-236.

Rizzolatti, G., \& Arbib, M. A. (1998). Language within our grasp. Trends in Neurosciences, 21, 188-194
Robertson, L. C., Lamb, M. R., \& Knight, R. T. (1988). Effects of lesions of temporal-parietal junction on perceptual and attentional processing in humans. Journal of Neuroscience, 8, 3757-3769.

Rorden, C., \& Brett, M. (2000). Stereotaxic display of brain lesions. Behavioural Neurology, 12, 191-200.

Rusconi, M. L., Maravita, A., Bottini, G., \& Vallar, G. (2002). Is the intact side really intact? Perseverative responses in patients with unilateral neglect: A productive manifestation. Neuropsychologia, 40, 594-604.

Russell, C., Malhotra, P., \& Husain, M. (2004). Attention modulates the visual field in healthy observers and parietal patients. NeuroReport, 15, 189-193.

Rypma, B., \& D'Esposito, M. (2003). A subsequent-memory effect in dorsolateral prefrontal cortex. Cognitive Brain Research, 16, 162-166.

Sapir, A., Hayes, A., Henik, A., Danzinger, S., \& Rafal, R. (2004). Parietal lobe lesions disrupt saccadic remapping of inhibitory location tagging. Journal of Cognitive Neuroscience, 16, 503-509.

Smania, N., Martini, M. C., Gambina, G. A. T., Palamara, A., Natale, E., \& Marzi, C. A. (1998). The spatial distribution of visual attention in hemineglect and extinction patients. Brain, 121, 1759-1770.

Sprenger, A., Kompf, D., \& Heide, W. (2002). Visual search in patients with left visual hemineglect. Progress in Brain Research, 140, 395-416.

Tobler, P., Felblinger, J., Burki, M., Nirkko, A., Ozdoba, C., \& Muri, R. (2001). Functional organisation of the saccadic reference system processing extraretinal signals in humans. Vision Research, 41, 1351-1358.

Wilson, B., Cockburn, J., \& Halligan, P. W. (1987). Behavioural inattention test. Bury St. Edmunds: Thames Valley.

Wojciulik, E., Husain, M., Clarke, K., \& Driver, J. (2001). Spatial working memory deficit in unilateral neglect. Neuropsychologia, 39, 390-396.

Zihl, J., \& Hebel, N. (1997). Patterns of oculomotor scanning in patients with unilateral posterior parietal and frontal lobe damage. Neuropsychologia, 35, 893-906.

Zihl, J., \& von Cramon, D. (1985). Visual field recovery from scotoma in patients with postgeniculate damage. A review of 55 cases. Brain, 108, 335-365. 